\title{
FIRST PASSAGE TIMES OF (REFLECTED) ORNSTEIN-UHLENBECK PROCESSES OVER RANDOM JUMP BOUNDARIES
}

\author{
LIJUN BO, ${ }^{*}$ Xidian University \\ YONGJIN WANG, ${ }^{* *}$ Nankai University \\ XUEWEI YANG, ${ }^{* * *}$ Nankai University and University of Illinois
}

\begin{abstract}
In this paper we study first passage times of (reflected) Ornstein-Uhlenbeck processes over compound Poisson-type boundaries. In fact, we extend the results of first rendezvous times of (reflected) Brownian motion and compound Poisson-type processes in Perry, Stadje and Zacks (2004) to the (reflected) Ornstein-Uhlenbeck case.

Keywords: First passage time; Ornstein-Uhlenbeck process; reflecting barrier; compound Poisson-type boundary

2010 Mathematics Subject Classification: Primary 60J60; 60K10
\end{abstract}

\section{Introduction}

First passage problems of (reflected) Ornstein-Uhlenbeck (OU) processes on a constant hitting level have been extensively investigated in Bo et al. (2006), (2010a), (2010b), (2011), Borovkov and Novikov (2008), Hadjiev (1985), Loeffen and Patie (2010), Patie (2005), and Alili et al. (2005). However, as far as we know, there is little literature on the study of first passage problems for (reflected) OU processes with respect to random hitting levels.

Recently, Perry et al. (2004) explored first passage times (FPTs) of (reflected) Brownian motion (BM) over compound Poisson-type boundaries. They found that the probability density function of the FPT admits an integral equation, and gave the probability density of the FPT for BM. Furthermore, the authors proved an explicit representation of the joint Laplace transform (LT) of the FPT and BM stopped at the FPT. In this paper we consider counterparts for (reflected) OU processes. We mainly extend all the results of first rendezvous times for the case of BM studied in Perry et al. (2004) to the (reflected) OU case. As in Perry et al. (2004), in order to obtain the joint LT for the (reflected) OU case, we have to calculate improper LTs of FPTs for (reflected) OU processes with constant boundaries and LTs evaluated at an exponential time (see Section 2 and Section 3 of the current paper). We find that the computations used in Perry et al. (2004) cannot be applied to the (reflected) OU case directly. To derive the abovementioned quantities, we need some other knowledge related to (reflected) OU processes, such as the transition density, the local time representation, and the density of the FPT with constant hitting levels, etc.

Received 2 November 2010; revision received 10 April 2011.

* Postal address: Department of Mathematics, Xidian University, Xi' an 710071, P. R. China.

** Postal address: School of Business, Nankai University, Tianjin 300071, P. R. China.

*** Postal address: School of Mathematical Sciences, Nankai University, Tianjin 300071, P. R. China.

Email address: xwyangnk@yahoo.com.cn 
In Section 2 we consider the FPT of OU processes over a one-jump boundary. In Section 3 we examine analogues for reflected OU (ROU) processes. The density of the FPT with a drifted-compound Poisson hitting level is discussed in Section 4.

\section{FPT of OU processes over a one-jump boundary}

It is known that the OU process satisfies the stochastic differential equation (SDE)

$$
\mathrm{d} X_{t}=\left(\mu-r X_{t}\right) \mathrm{d} t+\sigma \mathrm{d} W_{t},
$$

where the parameters $\mu \in \mathbb{R}$ and $r, \sigma>0$. Here $W=\left\{W_{t} ; t \geq 0\right\}$ is a standard Brownian motion on a complete filtered probability space $\left(\Omega, \mathcal{F}, \mathcal{F}_{t}, \mathrm{P}\right)$ with the filtration $\mathcal{F}_{t}$ satisfying the usual conditions. Moreover, the process $X=\left\{X_{t} ; t \geq 0\right\}$ is a strong Markov process with the following infinitesimal generator defined on $C_{b}^{2}(\mathbb{R})$ :

$$
\mathcal{A} f(x)=\frac{\sigma^{2}}{2} f^{\prime \prime}(x)+(\mu-r x) f^{\prime}(x) .
$$

We consider a random jump boundary $C_{1}=\left\{C_{1}(t) ; t \geq 0\right\}$ given by (see, e.g. Perry et al. (2004))

$$
C_{1}(t)=b+Y_{1} \mathbf{1}_{\left\{T_{1} \leq t\right\}},
$$

where the constant $b>0$ and the random variable $T_{1}$ admits an exponential law with parameter $q>0$, which is independent of the Brownian motion $W$. In addition, the random variable $Y_{1}$ is independent of $(T, W)$, which admits a distribution function given by $F(\mathrm{~d} y)$.

In this section we are concerned with the FPT defined by

$$
\tau_{0, b}=\inf \left\{t \geq 0: X_{t}=0 \text { or } X_{t}=C_{1}(t)\right\}
$$

where we set inf $\varnothing=\infty$ by convention. As usual, it is difficult to obtain an explicit expression for the joint distribution of the random vector $\left(X_{\tau_{0, b},}, \tau_{0, b}\right)$. Instead, we use the joint LT of the random vector $\left(X_{\tau_{0, b}, \tau_{0, b}}\right)$ to characterize the joint distribution. Let $\alpha, \theta>0$. Then we define

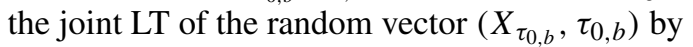

$$
\psi(\alpha, \theta ; x)=\mathrm{E}_{x}\left[\mathrm{e}^{-\alpha X_{\tau_{0, b}}-\theta \tau_{0, b}}\right],
$$

where $\mathrm{E}_{x}[\cdot]=\mathrm{E}\left[\cdot \mid X_{0}=x\right]$ for $0<x<b$, as usual. As in Perry et al. (2004), in order to obtain an explicit form for the joint LT (2.3), we have to compute the following quantities.

Improper LTs of FPTs for OU processes:

$$
\begin{aligned}
& \varphi_{1}(\theta ; b, x):=\mathrm{E}_{x}\left[\mathrm{e}^{-\theta \sigma_{0}} \mathbf{1}_{\left\{\sigma_{0}<\sigma_{b}\right\}}\right], \\
& \varphi_{2}(\theta ; b, x):=\mathrm{E}_{x}\left[\mathrm{e}^{-\theta \sigma_{b}} \mathbf{1}_{\left\{\sigma_{0}>\sigma_{b}\right\}}\right],
\end{aligned}
$$

where the stopping time $\sigma_{c}$ denotes the FPT of the OU process $X=\left\{X_{t} ; t \geq 0\right\}$ over a constant boundary $c \in \mathbb{R}$. That is, $\sigma_{c}=\inf \left\{t \geq 0: X_{t}=c\right\}$.

LTs evaluated at exponential time $T_{1}$ :

$$
\begin{aligned}
\phi_{0}(\alpha, \theta ; x) & :=\mathrm{E}_{x}\left[\mathrm{e}^{-\alpha X_{T_{1}}-\theta T_{1}}\right], \\
\phi_{1}(\alpha, \theta ; x) & :=\mathrm{E}_{x}\left[\mathrm{e}^{-\alpha X_{T_{1}}-\theta T_{1}} \mathbf{1}_{\left\{\tau_{0, b}<T_{1}\right\}}\right] .
\end{aligned}
$$


Before calculating the above quantities, we first present the following result concerning the LT of the FPT $\sigma_{c}$. For convenience, we define

$$
\gamma(\theta ; c, x)=\mathrm{E}_{x}\left[\mathrm{e}^{-\theta \sigma_{c}}\right]
$$

where $\theta>0$ and $c, x \in \mathbb{R}$. Clearly, $\gamma(0 ; c, x)=\gamma(\theta ; c, c)=1$. From Itô and McKean (1996, p. 130), we have (see also Alili et al. (2005)) the following result.

Lemma 2.1. For $x<c$, the LT (evaluated at $\theta>0$ ) of the FPT $\sigma_{c}$ is given by

$$
\gamma(\theta ; c, x)=\frac{f_{\theta}(x)}{f_{\theta}(c)},
$$

where $f_{\theta}(x)$ is the unique (up to a multiplicative constant) increasing positive solution of the equation

$$
\mathcal{A} f(x)=\theta f(x)
$$

with $\mathcal{A}$ given by (2.1).

Similarly as in Proposition 2.1 of Alili et al. (2005), the desired function $f_{\theta}(x)$ is

$$
f_{\theta}(x)=H_{-\theta / r}\left(-\frac{\sqrt{r}}{\sigma}\left(x-\frac{\mu}{r}\right)\right),
$$

with $H_{v}(x)$ being the Hermite function. Thus, by the symmetry of the process with respect to the long-run mean $\mu / r$, we have the following result.

Remark 2.1. The LT (evaluated at $\theta>0$ ) of the FPT $\sigma_{c}$ is given by

$$
\gamma(\theta ; c, x)= \begin{cases}\frac{H_{-\theta / r}(-\sqrt{r}(x-\mu / r) / \sigma)}{H_{-\theta / r}(-\sqrt{r}(c-\mu / r) / \sigma)} & \text { for } x<c, \\ \frac{H_{-\theta / r}(\sqrt{r}(x-\mu / r) / \sigma)}{H_{-\theta / r}(\sqrt{r}(c-\mu / r) / \sigma)} & \text { for } x>c .\end{cases}
$$

Now we can present the expressions for the improper LTs.

Lemma 2.2. Let $0<x<b$. The improper LTs are given by

and

$$
\varphi_{1}(\theta ; b, x)=\frac{f_{\theta}(x) \gamma(\theta ; 0, b)-f_{\theta}(b) \gamma(\theta ; 0, x)}{f_{\theta}(0) \gamma(\theta ; 0, b)-f_{\theta}(b)}
$$

$$
\varphi_{2}(\theta ; b, x)=\frac{f_{\theta}(0) \gamma(\theta ; 0, x)-f_{\theta}(x)}{f_{\theta}(0) \gamma(\theta ; 0, b)-f_{\theta}(b)},
$$

where the functions $f_{\theta}(x)$ and $\gamma(\theta ; b, x)$ are given in (2.5) and (2.6).

Proof. Since the function $f_{\theta}(x)$ is a solution to the ordinary differential equation (ODE) (2.4), we use Itô's formula and the optional time theorem to conclude that

$$
\mathrm{E}_{x}\left[\mathrm{e}^{-\theta\left(\sigma_{0} \wedge \sigma_{b}\right)} f_{\theta}\left(X_{\sigma_{0} \wedge \sigma_{b}}\right)\right]=f_{\theta}(x) .
$$

Hence, we have

$$
f_{\theta}(x)=f_{\theta}(0) \varphi_{1}(\theta ; b, x)+f_{\theta}(b) \varphi_{2}(\theta ; b, x),
$$


by employing the fact that $X_{\sigma_{c}}=c$ for all $c \in \mathbb{R}$. For the LT of the FPT $\sigma_{0}$, it holds that

$$
\gamma(\theta ; 0, x)=\varphi_{1}(\theta ; b, x)+\mathrm{E}_{x}\left[\mathrm{e}^{-\theta \sigma_{0}} \mathbf{1}_{\left\{\sigma_{b}<\sigma_{0}\right\}}\right] .
$$

For the second term on the right-hand side, using the strong Markov property of the OU process $X$ and the equality $\sigma_{0}=\sigma_{b}+\sigma_{0} \circ \zeta_{\sigma_{b}}$ on the event $\left\{\omega ; \sigma_{b}(\omega)<\sigma_{0}(\omega)\right\}$, where $\zeta$. is the shift operator, we obtain

$$
\begin{aligned}
\mathrm{E}_{x}\left[\mathrm{e}^{-\theta \sigma_{0}} \mathbf{1}_{\left\{\sigma_{b}<\sigma_{0}\right\}}\right] & =\mathrm{E}_{x}\left[\mathrm{E}_{x}\left[\mathrm{e}^{-\theta \sigma_{0}} \mathbf{1}_{\left\{\sigma_{b}<\sigma_{0}\right\}} \mid \mathcal{F}_{\sigma_{b}}\right]\right] \\
& =\mathrm{E}_{x}\left[\mathrm{e}^{-\theta \sigma_{b}} \mathbf{1}_{\left\{\sigma_{b}<\sigma_{0}\right\}} \mathrm{E}_{x}\left[\mathrm{e}^{-\theta\left(\sigma_{0} \circ \zeta_{\sigma_{b}}\right)} \mid \mathcal{F}_{\sigma_{b}}\right]\right] \\
& =\mathrm{E}_{x}\left[\mathrm{e}^{-\theta \sigma_{b}} \mathbf{1}_{\left\{\sigma_{b}<\sigma_{0}\right\}} \mathrm{E}_{X_{\sigma_{b}}}\left[\mathrm{e}^{-\theta \sigma_{0}}\right]\right] \\
& =\mathrm{E}_{x}\left[\mathrm{e}^{-\theta \sigma_{b}} \mathbf{1}_{\left\{\sigma_{b}<\sigma_{0}\right\}} \mathrm{E}_{b}\left[\mathrm{e}^{-\theta \sigma_{0}}\right]\right] \\
& =\gamma(\theta ; 0, b) \varphi_{2}(\theta ; b, x) .
\end{aligned}
$$

This yields the equality

$$
\gamma(\theta ; 0, x)=\varphi_{1}(\theta ; b, x)+\gamma(\theta ; 0, b) \varphi_{2}(\theta ; b, x) .
$$

Solving for $\varphi_{1}(\theta ; b, x)$ and $\varphi_{2}(\theta ; b, x)$ in (2.7) and (2.8), we arrive at the desired expressions for $\varphi_{1}(\theta ; b, x)$ and $\varphi_{2}(\theta ; b, x)$.

In Proposition 2.1 below we compute the LTs evaluated at the exponential time $T_{1}$. Note that we use the fact that, by l'Hôpital's rule,

$$
\lim _{\alpha \downarrow 0} \phi_{0}(\alpha, \theta ; x)=\frac{q}{r} \lim _{\alpha \downarrow 0} \frac{\int_{0}^{\alpha}\left(\mathrm{e}^{-x z-w(z)} / z\right) \mathrm{d} z}{e^{-w(\alpha)}}=-\frac{q}{r} \lim _{\alpha \downarrow 0} \frac{\mathrm{e}^{-x \alpha}}{\alpha w^{\prime}(\alpha)}=\frac{q}{q+\theta} .
$$

Proposition 2.1. Let $x \in \mathbb{R}$. We have, for $\alpha>0$,

$$
\phi_{0}(\alpha, \theta ; x)=\frac{q}{r} \int_{0}^{\alpha} \frac{\mathrm{e}^{w(\alpha)-x z-w(z)}}{z} \mathrm{~d} z
$$

with

$$
w(\alpha)=-\frac{\alpha \mu}{r}+\frac{\alpha^{2} \sigma^{2}}{4 r}-\frac{q+\theta}{r} \ln \alpha .
$$

Moreover, for $0<x<b$, we have

$$
\phi_{1}(\alpha, \theta ; x)=\phi_{0}(\alpha, \theta ; 0) \varphi_{1}(q+\theta ; b, x)+\phi_{0}(\alpha, \theta ; b) \varphi_{2}(q+\theta ; b, x),
$$

where $\varphi_{1}(q+\theta ; b, x)$ and $\varphi_{2}(q+\theta ; b, x)$ were given in Lemma 2.2.

Proof. By virtue of Itô's formula,

$$
M_{t}:=\mathrm{e}^{-\alpha X_{t}-\theta t}-\mathrm{e}^{-\alpha X_{0}}+\left(\alpha \mu+\theta-\frac{1}{2} \sigma^{2} \alpha^{2}\right) \int_{0}^{t} \mathrm{e}^{-\alpha X_{s}-\theta s} \mathrm{~d} s-\alpha r \int_{0}^{t} X_{s} \mathrm{e}^{-\alpha X_{s}-\theta s} \mathrm{~d} s
$$

is a martingale with mean 0 . As a consequence,

$$
\begin{aligned}
& \phi_{0}(\alpha, \theta ; x)+\left(\alpha \mu+\theta-\frac{1}{2} \sigma^{2} \alpha^{2}\right) \mathrm{E}_{x} \int_{0}^{T_{1}} \mathrm{e}^{-\alpha X_{s}-\theta s} \mathrm{~d} s-\alpha r \mathrm{E}_{x} \int_{0}^{T_{1}} X_{s} \mathrm{e}^{-\alpha X_{s}-\theta s} \mathrm{~d} s \\
& =\mathrm{e}^{-\alpha x} .
\end{aligned}
$$


Since the random variable $T_{1}$ has an exponential law with parameter $q>0$, which is independent of the OU process $X$, we have

$$
\mathrm{E}_{x} \int_{0}^{T_{1}} \mathrm{e}^{-\alpha X_{s}-\theta s} \mathrm{~d} s=\mathrm{E}_{x} \int_{0}^{\infty}\left(\int_{s}^{\infty} q \mathrm{e}^{-q t} \mathrm{~d} t\right) \mathrm{e}^{-\alpha X_{s}-\theta s} \mathrm{~d} s=\frac{1}{q} \phi_{0}(\alpha, \theta ; x) .
$$

Similarly, we obtain

$$
\mathrm{E}_{x} \int_{0}^{T_{1}} X_{s} \mathrm{e}^{-\alpha X_{s}-\theta s} \mathrm{~d} s=-\frac{1}{q} \frac{\partial \phi_{0}(\alpha, \theta ; x)}{\partial \alpha} .
$$

Thus, $\phi_{0}(\alpha, \theta ; x)$ solves the ODE

$$
\frac{\partial \phi_{0}(\alpha, \theta ; x)}{\partial \alpha}=-\frac{q+\theta+\alpha \mu-\sigma^{2} \alpha^{2} / 2}{\alpha r} \phi_{0}(\alpha, \theta ; x)+\frac{q}{\alpha r} \mathrm{e}^{-\alpha x},
$$

and is subject to the boundary condition

$$
\lim _{\alpha \downarrow 0} \phi_{0}(\alpha, \theta ; x)=\frac{q}{q+\theta} .
$$

We solve (2.9) with boundary condition (2.10) and arrive at the expression of $\phi_{0}(\alpha, \theta ; x)$ given in the proposition.

Next we derive the expression for $\phi_{1}(\alpha, \theta ; x)$. Using the strong Markov property of the OU process $X$ and the memoryless property of the exponential distribution, we obtain

$$
\begin{aligned}
\mathrm{E}_{x}\left[\mathrm{e}^{-\alpha X_{T_{1}}-\theta T_{1}} \mathbf{1}_{\left\{\tau_{0, b}<T_{1}, X_{\tau_{0, b}}=0\right\}}\right] \\
\quad=\mathrm{E}_{x}\left[\mathrm{E}_{x}\left[\mathrm{e}^{-\alpha X_{T_{1}}-\theta T_{1}} \mathbf{1}_{\left\{\tau_{0, b}<T_{1}, X_{\tau_{0, b}}=0\right\}} \mid \mathcal{F}_{\tau_{0, b}}\right]\right] \\
\quad=\mathrm{E}_{x}\left[\mathbf{1}_{\left\{\tau_{0, b}<T_{1}, X_{\tau_{0, b}}=0\right\}} \mathrm{E}_{x}\left[\mathrm{e}^{-\alpha X_{T_{1}}-\theta T_{1}} \mid \mathcal{F}_{\tau_{0, b}}\right]\right] \\
\left.\quad=\mathrm{E}_{x}\left[\mathrm{e}^{-\theta \tau_{0, b}} \mathbf{1}_{\left\{\tau_{0, b}<T_{1}, X_{\tau_{0, b}}=0\right\}} \mathrm{E}_{x} \mathrm{e}^{-\alpha X_{T_{1}} \circ \zeta_{\tau_{0, b}}-\theta T_{1} \circ \zeta_{\tau_{0, b}}} \mid \mathcal{F}_{\tau_{0, b}}\right]\right] \\
\quad=\phi_{0}(\alpha, \theta ; 0) \mathrm{E}_{x}\left[\mathrm{e}^{-\theta \tau_{0, b}} \mathbf{1}_{\left\{\tau_{0, b}<T_{1}, X_{\tau_{0, b}}=0\right\}}\right] .
\end{aligned}
$$

In addition, the expectation in the last line of (2.11) is given by

$$
\begin{aligned}
\mathrm{E}_{x}\left[\mathrm{e}^{-\theta \tau_{0, b}} \mathbf{1}_{\left\{\tau_{0, b}<T_{1}, X_{\tau_{0, b}}=0\right\}}\right] & =\mathrm{E}_{x}\left[\mathrm{e}^{-\theta\left(\sigma_{0} \wedge \sigma_{b}\right)} \mathbf{1}_{\left\{X_{\sigma_{0} \wedge \sigma_{b}}=0\right\}} \mathrm{P}\left(T_{1}>\sigma_{0} \wedge \sigma_{b} \mid X\right)\right] \\
& =\mathrm{E}_{x}\left[\mathrm{e}^{-(\theta+q)\left(\sigma_{0} \wedge \sigma_{b}\right)} \mathbf{1}_{\left\{X_{\sigma_{0} \wedge \sigma_{b}}=0\right\}}\right] \\
& =\varphi_{1}(q+\theta ; b, x),
\end{aligned}
$$

since the random variable $T_{1}$ has an exponential law with parameter $q$, which is independent of the OU process $X$, and the event $\left\{\omega ; X_{\sigma_{0}(\omega) \wedge \sigma_{b}(\omega)}(\omega)=0\right\}$ is identical to $\left\{\omega ; \sigma_{0}(\omega)<\sigma_{b}(\omega)\right\}$.

Similarly, it holds that

$$
\mathrm{E}_{x}\left[\mathrm{e}^{-\alpha X_{T_{1}}-\theta T_{1}} \mathbf{1}_{\left\{\tau_{0, b}<T_{1}, X_{\tau_{0, b}}=b\right\}}\right]=\phi_{0}(\alpha, \theta ; b) \varphi_{2}(q+\theta ; b, x) .
$$

Then the desired expression for $\phi_{1}(\alpha, \theta ; x)$ follows from the equality

$$
\phi_{1}(\alpha, \theta ; x)=\mathrm{E}_{x}\left[\mathrm{e}^{-\alpha X_{T_{1}}-\theta T_{1}} \mathbf{1}_{\left\{\tau_{0, b}<T_{1}, X_{\tau_{0, b}}=0\right\}}\right]+\mathrm{E}_{x}\left[\mathrm{e}^{-\alpha X_{T_{1}}-\theta T_{1}} \mathbf{1}_{\left\{\tau_{0, b}<T_{1}, X_{\tau_{0, b}}=b\right\}}\right] .
$$

This completes the proof. 
Remark 2.2. Clearly, the quantities $\phi_{0}(\alpha, \theta ; x)$ and $\phi_{1}(\alpha, \theta ; x)$ obtained in Proposition 2.1 together determine the joint distribution of the random vector $\left(X_{T_{1}}, T_{1}, \mathbf{1}_{\left\{T_{1}<\tau_{0, b}\right\}}\right)$ (see the illustration on page 1064 of Perry et al. (2004)).

Note that the definition of $f(\theta ; y, x)$ in Equation (3.11) of Perry et al. (2004) should be

$$
\begin{aligned}
f(\theta ; y, x) & =\mathrm{E}_{x}\left[\mathrm{e}^{-\alpha X(R(a+b+y))-\theta R(a+b+y)}\right] \\
& =\psi_{0}^{*}(\theta, x, a+b+y-x)+\mathrm{e}^{-\alpha(a+b+y)} \psi_{1}^{*}(\theta, x, a+b+y-x) .
\end{aligned}
$$

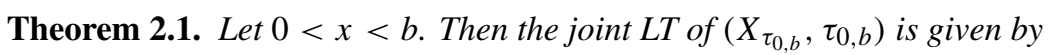

$$
\psi(\alpha, \theta ; x)=\varphi_{1}(q+\theta ; b, x)+\mathrm{e}^{-\alpha b} \varphi_{2}(q+\theta ; b, x)+\mathrm{E}_{x}\left[\mathrm{e}^{-\theta T_{1}} h\left(\theta ; X_{T_{1}}, Y_{1}\right) \mathbf{1}_{\left\{\tau_{0, b}>T_{1}\right\}}\right],
$$

where the function $h(\theta ; x, y)=\varphi_{1}(\theta ; b+y, x)+\mathrm{e}^{-\alpha(b+y)} \varphi_{2}(\theta ; b+y, x)$ for $y>0$. The third term on the right-hand side can be determined from Proposition 2.1.

\section{FPT of ROU processes over a one-jump boundary}

It is known that the ROU process on the state space $[0, \infty)$ satisfies the SDE

$$
\mathrm{d} \hat{X}_{t}=\left(\mu-r \hat{X}_{t}\right) \mathrm{d} t+\sigma \mathrm{d} W_{t}+\mathrm{d} L_{t},
$$

where the parameters $\mu \in \mathbb{R}$ and $r, \sigma>0$. The process $L=\left\{L_{t} ; t \geq 0\right\}$ is called a regulator at boundary 0 of the ROU process $\hat{X}=\left\{\hat{X}_{t} ; t \geq 0\right\}$ (see, e.g. Ata et al. (2005) for details). The regulator $L$ is continuous, nondecreasing, and satisfies the property that

$$
L_{t}=\int_{0}^{t} \mathbf{1}_{\left\{\hat{X}_{s}=0\right\}} \mathrm{d} L_{s} \quad \text { for all } t \geq 0 .
$$

It holds that $\hat{X}_{t} \geq 0$ for all $t \geq 0$ if the initial date $\hat{X}_{0} \geq 0$ (see, e.g. Ata et al. (2005) or Harrison (1985, p. 20) for details). In fact, the regulator $L$ is closely related to the local time at 0 of the ROU process $\hat{X}$, which will be stated in the following lemma.

Lemma 3.1. The regulator $L$ in (3.1) has the following two representations.

- Skorohod representation:

$$
L_{t}=\sup _{0 \leq s \leq t}\left(\hat{X}_{0}+\int_{0}^{s}\left(\mu-r \hat{X}_{u}\right) \mathrm{d} u+\sigma W_{s}\right)^{-},
$$

where $x^{-}:=\max \{-x, 0\}$ for $x \in \mathbb{R}($ see Karatzas and Shreve $(1991, p .210))$.

- Local time representation:

$$
L_{t}=\lim _{\varepsilon \rightarrow 0} \frac{\sigma^{2}}{2 \varepsilon} \int_{0}^{t} \mathbf{1}_{\left\{0 \leq \hat{X}_{s} \leq \varepsilon\right\}} \mathrm{d} s .
$$

Proof. Since the validity of (3.3) is clear, we only prove (3.4). Let $\ell^{a}=\left\{\ell_{t}^{a} ; t \geq 0\right\}$ denote the local time process of the ROU process $\hat{X}$ at point $a \geq 0$. Then, by virtue of Protter (2004, p. 225), the local time $\ell^{a}$ has the representation

$$
\ell_{t}^{a}=\lim _{\varepsilon \rightarrow 0} \frac{1}{\varepsilon} \int_{0}^{t} \mathbf{1}_{\left\{0 \leq \hat{X}_{s}-a \leq \varepsilon\right\}} \mathrm{d}[\hat{X}, \hat{X}]_{s}^{c}=\lim _{\varepsilon \rightarrow 0} \frac{\sigma^{2}}{\varepsilon} \int_{0}^{t} \mathbf{1}_{\left\{0 \leq \hat{X}_{s}-a \leq \varepsilon\right\}} \mathrm{d} s .
$$


On the other hand, using Meyer-Tanaka's formula (see, e.g. Protter (2004, p. 216)) for the ROU process $\hat{X}$ yields

$$
\begin{aligned}
\hat{X}_{t} & =\hat{X}_{0}+\int_{0}^{t} \mathbf{1}_{\left\{\hat{X}_{s}>0\right\}} \mathrm{d} \hat{X}_{s}-\int_{0}^{t} \mathbf{1}_{\left\{\hat{X}_{s}=0\right\}} \mathrm{d} \hat{X}_{s}+\ell_{t}^{0} \\
& =\hat{X}_{t}-2 \int_{0}^{t} \mathbf{1}_{\left\{\hat{X}_{s}=0\right\}} \mathrm{d} \hat{X}_{s}+\ell_{t}^{0} \\
& =\hat{X}_{t}-2 \int_{0}^{t} \mathbf{1}_{\left\{\hat{X}_{s}=0\right\}}\left(\mu-r \hat{X}_{s}\right) \mathrm{d} s-2 \sigma \int_{0}^{t} \mathbf{1}_{\left\{\hat{X}_{s}=0\right\}} \mathrm{d} W_{s}-2 \int_{0}^{t} \mathbf{1}_{\left\{\hat{X}_{s}=0\right\}} \mathrm{d} L_{s}+\ell_{t}^{0} \\
& =\hat{X}_{t}-2 \mu \int_{0}^{t} \mathbf{1}_{\left\{\hat{X}_{s}=0\right\}} \mathrm{d} s-2 \sigma \int_{0}^{t} \mathbf{1}_{\left\{\hat{X}_{s}=0\right\}} \mathrm{d} W_{s}-2 L_{t}+\ell_{t}^{0},
\end{aligned}
$$

where we have used the fact that $\hat{X}_{t} \geq 0$ for all $t \geq 0$ and property (3.2). As a consequence,

$$
L_{t}=-\mu \int_{0}^{t} \mathbf{1}_{\left\{\hat{X}_{s}=0\right\}} \mathrm{d} s-\sigma \int_{0}^{t} \mathbf{1}_{\left\{\hat{X}_{s}=0\right\}} \mathrm{d} W_{s}+\frac{1}{2} \ell_{t}^{0} .
$$

The above equality shows that $\int_{0}^{t} \mathbf{1}_{\left\{\hat{X}_{s}=0\right\}} \mathrm{d} W_{s}=0$, since a continuous local martingale of finite variation is equal to the initial value. So

$$
L_{t}=-\mu \int_{0}^{t} \mathbf{1}_{\left\{\hat{X}_{s}=0\right\}} \mathrm{d} s+\frac{1}{2} \ell_{t}^{0} .
$$

To complete the proof, we employ (3.5) with $a=0$ and note that $\int_{0}^{t} \mathbf{1}_{\left\{\hat{X}_{s}=0\right\}} \mathrm{d} s=0$ a.s.

We here point out that the explicit expression for the transition density $p(t ; x, y)$ of the ROU process $\hat{X}$ has been obtained in Linetsky (2005) (see Section 5.2 therein). Moreover, the explicit expression for the LT

$$
\hat{\gamma}(\theta ; b, x):=\mathrm{E}_{x}\left[\mathrm{e}^{-\theta \hat{\sigma}_{b}}\right]
$$

of the FPT $\hat{\sigma}_{c}=\inf \left\{t \geq 0: \hat{X}_{t}=c\right\}$ has been obtained in Theorem 2.1 of Bo et al. (2006) and Remark 4 of Bo et al. (2011). Hereafter, we will treat $p(t ; x, y)$ and $\hat{\gamma}(\theta ; b, x)$ as known functions.

In this section we are concerned with the following FPT of ROU process (3.1) over the one-jump boundary $C_{1}$ given by (2.2):

$$
\hat{\tau}_{b}=\inf \left\{t \geq 0: \hat{X}_{t}=C_{1}(t)\right\} .
$$

Here we set inf $\varnothing=\infty$ by convention. As in Perry et al. (2004), in order to derive an explicit form for the joint LT

$$
\hat{\psi}(\alpha, \theta ; x)=\mathrm{E}_{x}\left[\mathrm{e}^{-\alpha X_{\hat{\tau}_{b}}-\theta \hat{\tau}_{b}}\right],
$$

we have to compute the LTs evaluated at the exponential time $T_{1}$ :

$$
\begin{aligned}
& \hat{\phi}_{0}(\alpha, \theta ; x):=\mathrm{E}_{x}\left[\mathrm{e}^{-\alpha \hat{X}_{T_{1}}-\theta T_{1}}\right], \\
& \hat{\phi}_{1}(\alpha, \theta ; x):=\mathrm{E}_{x}\left[\mathrm{e}^{-\alpha \hat{X}_{T_{1}}-\theta T_{1}} \mathbf{1}_{\left\{\hat{\tau}_{b}<T_{1}\right\}}\right] .
\end{aligned}
$$

In fact, we have the following proposition. 
Proposition 3.1. Let $0<x<b$. We have

$$
\hat{\phi}_{0}(\alpha, \theta ; x)=\frac{q}{r} \int_{0}^{\alpha} \frac{\mathrm{e}^{w(\alpha)-x z-w(z)}}{z} \mathrm{~d} z+\frac{\sigma^{2}}{2 r} \hat{g}_{L}(\theta ; x) \int_{0}^{\alpha} \mathrm{e}^{w(\alpha)-w(z)} \mathrm{d} z \quad \text { for } \alpha>0,
$$

with $w(\alpha)$ defined in Proposition 2.1. Here the function $\hat{g}_{L}(\theta ; x)=\mathrm{E}_{x}\left[\mathrm{e}^{-\theta T_{1}} p\left(T_{1} ; x, 0\right)\right]$, where $p(t ; x, 0)$ is the transition density at $y=0$ of the ROU process $\hat{X}$. Moreover,

$$
\hat{\phi}_{1}(\alpha, \theta ; x)=\hat{\phi}_{0}(\alpha, \theta ; b) \hat{\gamma}(q+\theta ; b, x),
$$

where the $L T \hat{\gamma}(q+\theta ; b, x)$ is defined in (3.6).

Proof. By virtue of Itô's formula,

$$
\begin{aligned}
\hat{M}_{t}:= & \mathrm{e}^{-\alpha \hat{X}_{t}-\theta t}-\mathrm{e}^{-\alpha \hat{X}_{0}}+\left(\alpha \mu+\theta-\frac{1}{2} \sigma^{2} \alpha^{2}\right) \int_{0}^{t} \mathrm{e}^{-\alpha \hat{X}_{s}-\theta s} \mathrm{~d} s \\
& -\alpha r \int_{0}^{t} \hat{X}_{s} \mathrm{e}^{-\alpha \hat{X}_{s}-\theta s} \mathrm{~d} s+\alpha \int_{0}^{t} \mathrm{e}^{-\alpha \hat{X}_{s}-\theta s} \mathrm{~d} L_{s}
\end{aligned}
$$

is a martingale with mean 0 . Using (3.2), we obtain

$$
\begin{aligned}
& \hat{\phi}_{0}(\alpha, \theta ; x)+\left(\alpha \mu+\theta-\frac{1}{2} \sigma^{2} \alpha^{2}\right) \mathrm{E}_{x} \int_{0}^{T_{1}} \mathrm{e}^{-\alpha \hat{X}_{s}-\theta s} \mathrm{~d} s-\alpha r \mathrm{E}_{x} \int_{0}^{t} \hat{X}_{s} \mathrm{e}^{-\alpha \hat{X}_{s}-\theta s} \mathrm{~d} s \\
& +\alpha \mathrm{E}_{x} \int_{0}^{t} \mathrm{e}^{-\theta s} \mathrm{~d} L_{s}=\mathrm{e}^{-\alpha x} .
\end{aligned}
$$

Similarly as the proof of Proposition $2.1, \hat{\phi}_{0}(\alpha, \theta ; x)$ admits the ODE

$$
\frac{\partial \hat{\phi}_{0}(\alpha, \theta ; x)}{\partial \alpha}=-\frac{q+\theta+\alpha \mu-\sigma^{2} \alpha^{2} / 2}{\alpha r} \hat{\phi}_{0}(\alpha, \theta ; x)+\frac{q}{\alpha r} \mathrm{e}^{-\alpha x}+\frac{q}{r} g_{L}(\theta ; x)
$$

with the boundary condition $\lim _{\alpha \downarrow 0} \hat{\phi}_{0}(\alpha, \theta ; x)=q /(q+\theta)$, where the function $g_{L}(\theta ; x)$ is defined by

$$
g_{L}(\theta ; x)=\mathrm{E}_{x} \int_{0}^{T_{1}} \mathrm{e}^{-\theta s} \mathrm{~d} L_{s} .
$$

Thanks to Lemma 3.1, we can conclude that

$$
\begin{aligned}
g_{L}(\theta ; x) & =\frac{q}{2} \sigma^{2} \int_{0}^{\infty} \mathrm{e}^{-q t}\left(\int_{0}^{t} \mathrm{e}^{-\theta s} \lim _{\varepsilon \rightarrow 0} \frac{1}{\varepsilon} \mathrm{P}_{x}\left(\hat{X}_{s} \leq \varepsilon\right) \mathrm{d} s\right) \mathrm{d} t \\
& =\frac{q}{2} \sigma^{2} \int_{0}^{\infty} \mathrm{e}^{-q t}\left(\int_{0}^{t} \mathrm{e}^{-\theta s} \lim _{\varepsilon \rightarrow 0} \frac{1}{\varepsilon} \int_{0}^{\varepsilon} p(s ; x, y) \mathrm{d} y \mathrm{~d} s\right) \mathrm{d} t \\
& =\frac{q}{2} \sigma^{2} \int_{0}^{\infty} \mathrm{e}^{-q t}\left(\int_{0}^{t} \mathrm{e}^{-\theta s} p(s ; x, 0) \mathrm{d} s\right) \mathrm{d} t \\
& =\frac{\sigma^{2}}{2 q} \mathrm{E}_{x}\left[\mathrm{e}^{-\theta T_{1}} p\left(T_{1} ; x, 0\right)\right] .
\end{aligned}
$$

Solving (3.8) yields the expression for $\hat{\phi}_{0}(\alpha, \theta ; x)$ given in this proposition. 
Next we derive the expression for $\hat{\phi}_{1}(\alpha, \theta ; x)$. Using the strong Markov property of the ROU process $\hat{X}$ and the memoryless property of the exponential distribution, we have

$$
\begin{aligned}
\hat{\phi}_{1}(\alpha, \theta ; x) & =\mathrm{E}_{x}\left[\mathrm{e}^{-\alpha \hat{X}_{T_{1}}-\theta T_{1}} \mathbf{1}_{\left\{\hat{\tau}_{b}<T_{1}\right\}}\right] \\
& =\mathrm{E}_{x}\left[\mathrm{E}_{x}\left[\mathrm{e}^{-\alpha \hat{X}_{T_{1}}-\theta T_{1}} \mathbf{1}_{\left\{\hat{\tau}_{b}<T_{1}\right\}} \mid \mathcal{F}_{\hat{\tau}_{b}}\right]\right] \\
& =\mathrm{E}_{x}\left[\mathbf{1}_{\left\{\hat{\tau}_{b}<T_{1}\right\}} \mathrm{E}_{x}\left[\mathrm{e}^{-\alpha \hat{X}_{T_{1}}-\theta T_{1}} \mid \mathcal{F}_{\hat{\tau}_{b}}\right]\right] \\
& =\mathrm{E}_{x}\left[\mathrm{e}^{-\theta \hat{\tau}_{b}} \mathbf{1}_{\left\{\hat{\tau}_{b}<T_{1}\right\}} \mathrm{E}_{x}\left[\mathrm{e}^{-\alpha \hat{X}_{T_{1}} \circ \zeta_{\hat{\tau}_{b}}-\theta T_{1} \circ \zeta_{\hat{\tau}_{b}}} \mid \mathcal{F}_{\hat{\tau}_{b}}\right]\right] \\
& =\hat{\phi}_{0}(\alpha, \theta ; b) \mathrm{E}_{x}\left[\mathrm{e}^{-\theta \hat{\tau}_{b}} \mathbf{1}_{\left\{\hat{\tau}_{b}<T_{1}\right\}}\right] .
\end{aligned}
$$

In addition, the expectation in the last line of (3.10) is given by

$$
\mathrm{E}_{x}\left[\mathrm{e}^{-\theta \hat{\tau}_{b}} \mathbf{1}_{\left\{\hat{\tau}_{b}<T_{1}\right\}}\right]=\mathrm{E}_{x}\left[\mathrm{e}^{-\theta \hat{\sigma}_{b}} \mathrm{P}\left(T_{1}>\hat{\sigma}_{b} \mid \hat{X}\right)\right]=\hat{\gamma}(q+\theta ; b, x),
$$

since the random variable $T_{1}$ has an exponential law with parameter $q$, which is independent of the ROU process $\hat{X}$. This completes the proof.

Finally, we have the following result.

Theorem 3.1. Let $0<x<b$. Then the joint LT of $\left(\hat{X}_{\hat{\tau}_{b}}, \hat{\tau}_{b}\right)$ is given by

$$
\hat{\psi}(\alpha, \theta ; x)=\mathrm{e}^{-\alpha b} \hat{\gamma}(q+\theta ; b, x)+\mathrm{e}^{-\alpha b} \mathrm{E}_{x}\left[\mathrm{e}^{-\alpha Y_{1}-\theta T_{1}} \hat{\gamma}\left(\theta ; b+Y_{1}, \hat{X}_{T_{1}}\right) \mathbf{1}_{\left\{\hat{\tau}_{b}>T_{1}\right\}}\right],
$$

where the second term on the right-hand side can be determined from Proposition 3.1.

\section{FPT density of ROU processes over a compound Poisson process}

In this section we consider the following FPT of ROU process (3.1) over a boundary of drifted-compound Poisson process:

$$
\tilde{\tau}_{b}=\inf \left\{t \geq 0: \hat{X}_{t}=C(t)\right\} .
$$

Here $\hat{X}=\left\{\hat{X}_{t} ; t \geq 0\right\}$ is the ROU process (3.1) and the random boundary $C=\{C(t) ; t \geq 0\}$ is given by

$$
C(t)=b+\sum_{k=1}^{\infty} Y_{k} \mathbf{1}_{\left\{T_{k} \leq t\right\}},
$$

where $\left\{Y_{1}, Y_{2}, \ldots\right\}$ are independent and identically distributed random variables with probability density function (PDF) $f(y)$ and $T_{1}<T_{2}<\cdots<T_{n}<\cdots$ are jump times of the Poisson process $N=\left\{N_{t} ; t \geq 0\right\}$ with parameter $q>0$. In addition, $\left\{Y_{1}, Y_{2}, \ldots\right\},\left\{T_{1}, T_{2}, \ldots\right\}$, and BM $W$ are mutually independent.

Similarly to that of Theorem 2.1 of Perry et al. (2004), if the PDF $\hat{\rho}(t ; b)$ of the FPT $\hat{\sigma}_{b}$ for ROU process (3.1) is given, then the PDF $\tilde{\rho}(t ; b)$ of the FPT $\tilde{\tau}_{b}$ is the unique solution to the integral equation

$$
\tilde{\rho}(t ; b)=\mathrm{e}^{-q t} \hat{\rho}(t ; b)+\sum_{n=1}^{\infty} \int_{0}^{\infty} \int_{0}^{t} \mathrm{e}^{-q s} \frac{(q s)^{n}}{n !} f^{(n)}(z) \hat{\rho}(s ; b) \tilde{\rho}(t-s ; z) \mathrm{d} s \mathrm{~d} z,
$$

where $f^{(n)}(y)$ denotes the $n$-fold convolution of $f$ with itself.

Finally, we mention that the PDF $\hat{\rho}(t ; b)$ of the FPT $\hat{\sigma}_{b}$ for ROU process (3.1) has been obtained in terms of the spectral representation in Li et al. (2010, Proposition 2.1). 


\section{Acknowledgements}

The authors would like to thank an anonymous referee for valuable comments and suggestions which helped to improve the paper. This work was supported by the Keygrant Project of the Chinese Ministry of Education (grant number 309009) and the NSF of China (grant number 11001213).

\section{References}

Alili, L., Patie, P. and Pedersen, J. L. (2005). Representations of the first hitting time density of an OrnsteinUhlenbeck process. Stoch. Models 21, 967-980.

Ata, B., Harrison, J. M. And Shepp, L. A. (2005). Drift rate control of a Brownian processing system. Ann. Appl. Prob. 15, 1145-1160.

Bo, L., WANG, Y. AND YANG, X. (2010). First passage problems on reflected generalized Ornstein-Uhlenbeck processes and applications. Preprint.

Bo, L., WANG, Y. AND YANG, X. (2011). Some integral functionals of reflected SDEs and their applications in finance. Quant. Finance 11, 343-348.

Bo, L., ZHANG, L. AND WANG, Y. (2006). On the first passage times of reflected OU processes with two-sided barriers. Queueing Systems 54, 313-316.

Bo, L., TANG, D., WANG, Y. AND YANG, X. (2011). On the conditional default probability in a regulated market: a structural approach. Quant. Finance, 8pp.

Borovkov, K. And Novikov, A. (2008). On exit times of Levy-driven Ornstein-Uhlenbeck processes. Statist. Prob. Lett. 78, 1517-1525.

HadjIEv, D. I. (1985). The first passage problem for generalized Ornstein-Uhlenbeck processes with non-positive jumps. In Séminaire de Probabilités XIX (Lecture Notes Math. 1123), Springer, Berlin, pp. 80-90.

Harrison, J. M. (1985). Brownian Motion and Stochastic Flow Systems. John Wiley, New York.

Itô, K. And McKean, H. P., JR. (1996). Diffusion Processes and Their Sample Paths. Springer, Berlin.

Karatzas, I. And Shreve, S. E. (1991). Brownian Motion and Stochastic Calculus. Springer, New York.

LI, Y., WANG, Y. AND YANG, X. (2010). On the hitting time density for reflected OU processes: with an application to the regulated market. Preprint.

Linetsky, V. (2005). On the transition densities for reflected diffusions. Adv. Appl. Prob. 37, 435-460.

Loeffen, R. L. And Patie, P. (2010). Absolute ruin in the Ornstein-Uhlenbeck type risk model. Preprint. available at http://arxiv.org.abs/1006.2712v1.

Patie, P. (2005). On a martingale associated to generalized Ornstein-Uhlenbeck processes and an application to finance. Stoch. Process. Appl. 115, 593-607.

Perry, D., Stadje, W. And Zacks, S. (2004). The first rendezvous time of Brownian motion and compound Poissontype processes. J. Appl. Prob. 41, 1059-1070.

Protter, P. E. (2004). Stochastic Integration and Differential Equations. Springer, 